\title{
THE GROWTH OF POWERS OF A NONNEGATIVE MATRIX*
}

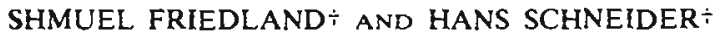

\begin{abstract}
Let $A$ be a nonnegative $n \times n$ matrix. In this paper we study the growth of the powers $A^{m}$, $m=1,2,3, \cdots$ when $\rho(A)=1$. These powers occur naturally in the iteration process$$
x^{(m-1)}=A x^{(m)}, \quad x^{(0)} \geq 0,
$$

which is important in applications and numerical techniques. Roughly speaking, we analyze the asymptotic behavior of each entry of $A^{m}$. We apply our main result to determine necessary and sufficient conditions for the convergence to the spectral radius of $A$ of certain ratios naturally associated with the iteration above.
\end{abstract}

\section{Introduction. Let $A$ be a nonnegative $n \times n$ matrix. In the iteration process}

$$
x^{(m+1)}=A x^{(m)}, \quad x^{(0)} \geqq 0,
$$

which is important in applications and numerical techniques, the powers $A^{m}, m=1$, $2, \cdots$ occur naturally. In this paper, we study the growth of these powers. In the literature there are several studies of the growth of $A^{m}$ when the elementary divisors belonging to the spectral radius $\rho(A)$ of $\dot{A}$ are linear. For example, see Gantmacher [7, Chap. 13, \$ 5-7] Varga [19, pp. 32-34] when $A$ is irreducible, and Meyer-Plemmons [10] when $\lim _{m \rightarrow \infty} A^{m}$ exists. We deal here with the general nonnegative case, when the elementary divisors belonging to $\rho(A)$ may have degrees greater than 1 . At the cost of ignoring nilpotent $A$, where the problem is trivial, we assume that $\rho(A)>0$.

For a complex $n \times n$ matrix $A$, with $\rho(A)=1$, there is a least integer $k$ for which $m^{-k} A^{m}$ is bounded, $m=1,2, \cdots$. However, even in the simple case of an imprimitive, irreducible nonnegative $A, \lim _{m \rightarrow \infty}\left\|m^{-k} A^{m}\right\|$ and, a fortiori $\lim _{m \rightarrow \infty} m^{-k} A^{\prime \prime}$, do not in general exist. To obtain precise results for general nonnegative $A$ with $\rho(A)=1$, it is thus necessary to introduce some smoothing. For example, in [14] Rothblum considered Cesaro means of powers of $A$. In this paper we study the growth of

$$
B^{(m)}=A^{m}\left(I+\cdots+A^{q-1}\right), \quad m=1,2, \cdots,
$$

where $q$ is a certain positive integer.

After some preliminaries in $\S 2$, we use elementary analytic methods in $\S 3$ to prove a theorem on the growth of $B^{(m)}$. As corollary, we obtain a known theorem on the index of the eigenvalue 1. of $A$, cf. Schaefer [17, Chap. 1, Thm. 2.7]. We also give a local form of the theorem; that is, we show that for $1 \leqq i, j \leqq n$ there exist integers $k=k(i, j)$ and $q=q(i, j)>0$ such that the element $b_{i j}^{(m)}$ of the matrix given by (1.2) satisfies

$$
\lim _{m \rightarrow \infty} m^{-k} b_{i j}^{(m)}>0
$$

The analytic results of $\S 3$ motivate the investigations in the rest of the paper.

The main thrust of the paper is the use of the graph structure of the matrix $A$ to decrease the integer $q(i, j)$ and to determine the integer $k(i, j)$ in (1.3). The requisite graph theoretic concepts are developed in $\S 4$, and in $\S 5$ we state our main result, Theorem (5.10). As a corollary, we obtain a striking theorem on the index of 1 due to

* Received by the editors June 14, 1979, and in revised form December 4, 1979.

+ Mathematics Department and Mathematics Research Center, University of Wisconsin, Madison. Wisconsin 53706. This research was supported in part by the United States Army under Contract DAAG29-75-C-0024 and by the National Science Foundation under Grant MCS78-01087. 
Rothblum [13]. Our results are related to those of U. G. Rothblum [14], [15], and in some instances, would also follow from his. But where Rothblum considers $A^{q \dot{m}}, m=$ $1,2, \cdots$, we consider $B^{(m)}$ and this allows us to choose a smaller integer $q$. Our definitions of $q(i, j)$ involves the greatest common divisor (g.c.d.) of certain periods where one might expect the least common multiple (I.c.m.). Consider the example

$$
A=\left[\begin{array}{lllll}
0 & 1 & 0 & 0 & 1 \\
1 & 0 & 0 & 0 & 0 \\
0 & 0 & 0 & 1 & 0 \\
0 & 0 & 0 & 0 & 1 \\
0 & 0 & 1 & 0 & 0
\end{array}\right]
$$

Then, by direct computation, for $1 \leqq i, j \leqq 2, \lim _{m \rightarrow \infty} b_{i j}^{(m)}=1$, where $B^{(m)}=$ $A^{m}(I+A)$. Thus $k(i, j)=0$, and we may choose $q(i, j)=2$ if $1 \leqq i, j \leqq 2$. Similarly $k(i, j)=0, q(i, j)=3$ if $3 \leqq i, j \leqq 5$. Yet $\lim _{m \rightarrow \infty} m^{-1} a_{i j}^{(m)}=\frac{1}{6}$ if $1 \leqq i \leqq 2,3 \leqq j \leqq 6$, and so we have $k(i, j)=1, q(i, j)=1$. We might add that it may be possible that our choice of $q(i, j)$ can be improved in the general case where we use an l.c.m. of certain g.c.d.'s.

In $\S 6$, we apply our results to the iteration process (1.1) for any nonnegative matrix $A$ satisfying $\rho(A)>0$. For $x \geqq 0, x \neq 0$ denote

$$
\begin{aligned}
& r(x)=\sup \{\mu: \mu x \leqq A x\}, \\
& R(x)=\inf \{\mu: \mu x \geqq A x\} .
\end{aligned}
$$

In Theorem 6.8, we find necessary and sufficient conditions for $r\left(A^{m} x\right)$ and $R\left(A^{m} x\right)$ to converge to the spectral radius of $A$. We show that whether or not this happens depends only on what is in general a small part of the vector $x$. In $\S 7$, we show that a theorem due to D. H. Carlson [3] on the existence of nonnegative solutions $y$ for $(I-A) y=x, x \geqq 0$, $\rho(A)=1$ is a consequence of our main results and we extend the theorem.

\section{Preliminaries.}

Notations. Let $\varphi(1), \varphi(2), \cdots$, be a sequence of nonnegative numbers and $k \geqq 0$ be an integer.

(i) $\varphi(m)=O\left(m^{k}\right)$ will denote that $\varphi(m) / m^{k}, m=1,2, \cdots$, is bounded.

(ii) $\varphi(m)=o\left(m^{k}\right)$ will denote that $\lim _{m \rightarrow \infty} \varphi(m) / m^{k}=0$.

(iii) $\varphi(m) \approx m^{k}$ will denote that $\lim _{m \rightarrow \infty} \varphi(m) / m^{k}$ exists and is positive.

(iv) The above notations will also be used for $k=-1,-\infty$. In case that $k=-1$ $\varphi(m)=O\left(m^{k}\right), \varphi(m)=o\left(m^{k}\right), \varphi(m) \approx m^{k}$ will each indicate that there exists $\rho, 0<\rho<1$, such that $\varphi(m) \rho^{-m}=O(1)$. In case that $k=-\infty$ the above notations will mean that $\varphi(m)=0$ for all sufficiently large $m$. (Thus $\varphi(m) \approx m^{-\infty}$ implies $\varphi(m) \approx m^{-1}$.)

(v) The notation $\boldsymbol{A}(m) \approx m^{k}$ will be used for a sequence of nonnegative matrices $\boldsymbol{A}(1), \boldsymbol{A}(2), \cdots$ to indicate the relation holds for each element.

Combinatorial result. Let $r \geqq 0$ and $t>0$ be integers. Then

$$
\Gamma_{t}^{r}=\sum_{p_{1}+\cdots+p_{t}=r} 1^{p_{1}} 1^{p_{2}} \cdots 1^{p_{t}},
$$

where the summation is taken over all nonnegative integers $p_{1}, \cdots, p_{r}$ whose sum is $r$. That is, $\Gamma^{r}$ is the number of collections of $r$ objects chosen from $t$ distinct objects, with 
repetitions allowed. It is well-known that

$$
\Gamma_{t}^{r}=\left(\begin{array}{c}
r+t-1 \\
r
\end{array}\right)
$$

A simple way to prove this equality is by considering the coefficient of $x^{r}$ of both sides of the identity

$$
\sum_{r=0}^{\infty}\left(\begin{array}{c}
r+t-1 \\
r
\end{array}\right) x^{r}=\left(\sum_{r=0}^{\infty} x^{r}\right)^{-t}
$$

which is derived from $(1-x)^{-t}=(1-x)^{-1} \cdots(1-x)^{-1}$. For a purely combinatorial proof see for example Brualdi [2, p. 37]. For $t=0$ the above formula implies $\Gamma_{0}^{\prime}=1$ for all $r \geqq 0$.

We shall also need some results on the convergence of series.

LEMMA 2.4. Given integers $k \geqq 1, q>0$, and let $b_{p} \geqq 0, p=0,1,2, \cdots$ be a sequence such that

$$
\lim _{p \rightarrow \infty} p^{-(k-1)}\left(b_{p}+\cdots+b_{p+q-1}\right)=v
$$

where $q>0$. Then

$$
\lim _{m \rightarrow \infty} m^{-k} \sum_{p=1}^{m} b_{p}=\frac{v}{k q} .
$$

Proof. Elementary. Alternatively, check that $c_{m, p}=m^{-k} k p^{k-1}$ satisfies the assumptions of Hardy [8, Thm. 2, p. 43].

LEMMA 2.7. Suppose (2.5) holds. If $\lim _{m \rightarrow \infty} a_{m}=u$ then

$$
\lim _{m \rightarrow \infty} m^{-k} \sum_{p=1}^{m} a_{p} b_{m-p}=\frac{u v}{k q} .
$$

Proof. According to Hardy [8, Thm. 16, p. 64]

$$
\lim _{m \rightarrow \infty} \frac{\sum_{p=1}^{m} a_{p} b_{m-p}}{\sum_{p=1}^{m} b_{p}}=u
$$

since

$$
0 \leqq \frac{b_{m}}{\sum_{p=1}^{m} b_{p}} \leqq \frac{b_{m}+\cdots+b_{m+q-1}}{\sum_{p=1}^{m} b_{p}} \leqq \frac{2 v m^{(k-1)}}{v(2 k q)^{-1} m^{k}},
$$

and the last expression tends to 0 . If we apply (2.6) to (2.9) we obtain (2.8).

3. Analytic approach. By $\mathbb{R}$, resp. $\mathbb{C}$, we denote the real, resp. complex field, and by $\mathbb{R}_{+}$the nonnegative numbers. The set of real, resp. complex, nonnegative $r \times n$ matrices will be denoted by $\mathbb{R}^{\prime n}$, resp. $\mathbb{C}^{r n}, \mathbb{R}_{+}^{r n}$. We also write $A \geqq 0$ for $A \in \mathbb{R}_{+}^{r n}(A$ is nonnegative $)$ and $A>0$ when $A$ is positive $\left(a_{i j}>0, i=1, \cdots, r, j=1, \cdots, n\right)$.

Let $A \in \mathbb{C}^{n n}$. By $\operatorname{spec} A$ we denote the set of eigenvalues of $A$. Suppose that $\operatorname{spec} A=\left\{\lambda_{1}, \cdots, \lambda_{r}\right\}$, where the $\lambda_{\alpha}$ are pairwise distinct. It is known (cf. Gantmacher $\left[7\right.$, Chap. 5, §3]) that there exist nonnegative integers $p_{1}, \cdots, p_{r}$ and unique matrices $Z^{(\alpha \beta)} \in \mathbb{C}^{n n}, \beta=0, \cdots, p_{\alpha}, \alpha=1, \cdots, r$ which are linearly independent such that for each polynomial $f(\tau)$,

$$
f(A)=\sum_{\alpha=1}^{r} \sum_{\beta=0}^{p_{\alpha}} f^{(\beta)}(\lambda) Z^{(\alpha \beta)}
$$


The $Z^{(\alpha \beta)}$ are polynomials in $A, p_{\alpha}+1$ is the size of a largest Jordan-block belonging to $\lambda_{\alpha}$. The columns of $Z^{\left(\alpha p_{\alpha}\right)}$ are eigenvectors of $A$ corresponding to the eigenvalue $\lambda_{\alpha}$, the rank of $Z^{\alpha p_{\alpha}}$ is equal to the number of Jordan blocks of size $p_{\alpha}+1$ corresponding to $\lambda_{\alpha}$. (The simplest way to obtain (3.1) is by assuming that $A$ is in Jordan form.) As usual we define

$$
\operatorname{index}\left(\lambda_{\alpha}\right)=p_{\alpha}+1 \text {. }
$$

That is, $p_{\alpha}+1$ is the multiplicity of $\lambda_{\alpha}$ in the minimal polynomial of $A$. We shall also use a localized index. For $1 \leqq i, j \leqq n$ we put

$$
\operatorname{index}_{i j}\left(\lambda_{\alpha}\right)=1+\max \left\{\beta: z_{i i}^{(\alpha \beta)} \neq 0, \beta=0, \cdots, p_{\alpha}\right\} \text {, }
$$

where index $x_{i j}\left(\lambda_{\alpha}\right)=0$ if $z_{i j}^{(\alpha \beta)}=0, \beta=0, \cdots, p_{\alpha}$. If $A \in \mathbb{C}^{n n}$ and $m$ is any integer we shall denote the elements of $A^{m}$ by $a_{i j}^{(m)}, 1 \leqq i, j \leqq m$.

Let $A \in \mathbb{R}_{+}^{n n}$. We assume throughout the normalization $\rho(A)=1$. It is well-known (see Frobenius [6], Gantmacher [7, Chap. 13], Berman-Plemmons [1, Chap. 2]) that if $\lambda$ is an eigenvalue of $A$ and $|\lambda|=1$, then $\lambda$ is a root of 1 . Hence, there is a positive integer $q$ such that $\lambda^{q}=1$, for all $\lambda \in \operatorname{spec} A,|\lambda|=1$. The smallest such integer $q$ will be called the period of $A$. If $q=1, A$ will be called aperiodic. For an irreducible and aperiodic matrix $A \geqq 0$, the Frobenius theorem and the formula (3.1) imply

$$
\lim _{m \rightarrow \infty} A^{m}=Z^{(10)}>0
$$

where $\lambda_{1}=1$, see for example Berman-Plemmons [1, Chap. 2, Thm. 4.1]. Theorem 3.4 extends the above equality in a local way. Part (i) of the theorem is an extension of the known inequality apparently due to Schaefer [16, Thm. 2.4, p. 264],

$$
\operatorname{index}(\lambda) \leqq \operatorname{index}(1) \text { if }|\lambda|=1 \text {, }
$$

for nonnegative matrices; see also Schaefer [17, Chap. 1, Thm. 2.7], Berman-Plemmons [1, Chap. 1, Thm. 3.2]. This result and part (i) of Theorem 3.4 could easily be deduced from the classical Pringsheim theorem on analytic functions; e.g., see Titchmarsh [18, p. 214]. The use of the Pringsheim theorem in analyzing the spectral properties of nonnegative matrices can be traced back to Ostrowski [11] (see also Karlin [9] and Schaefer [16, Appendix] for the infinite dimensional case). See Friedland [5] for a detailed analysis of the Pringsheim theorem for rational functions which has certain analogs to the Frobenius theorem. For sake of completeness we bring a short and elementary independent proof of Theorem 3.4. To do so we need an easy lemma which probably is known.

LEMMA 3.3. Let $\lambda_{\alpha}, z_{\alpha}, \alpha=1, \cdots, r$ be complex numbers, where the $\lambda_{\alpha}$ are pairwise distinct. If $\lim _{m \rightarrow \infty}\left(\sum_{\alpha=1}^{r} \lambda_{\alpha}^{m} z_{\alpha}\right)$ exists, then $z_{\alpha}=0$ if $\left|\lambda_{\alpha}\right| \geqq 1, \lambda_{\alpha} \neq 1$.

Proof. Since $\lim _{m \rightarrow \infty} \lambda_{\alpha}^{m}$ exists for $\left|\lambda_{\alpha}\right|<1$ or $\lambda_{\alpha}=1$, without loss of generality we may assume that $\left|\lambda_{\alpha}\right| \geqq 1, \lambda_{\alpha} \neq 1, \alpha=1, \cdots, r$. Put $z=\left(z_{1}, \cdots, z_{r}\right)^{\prime} \in \mathbb{C}^{\prime}$ and $u^{(m)}=$ $\left(u_{m}, \cdots, u_{m+r-1}\right)^{t}$, where $u_{m}=\sum_{\alpha=1}^{r} \lambda_{\alpha}^{m} z_{\alpha}$. Let $\Lambda=\operatorname{diag}\left\{\lambda_{1}, \cdots, \lambda_{r}\right\} \in \mathbb{C}^{r r}$ and let $V=$ $\left(v_{\alpha \beta}\right)_{1}^{r} \in \mathbb{C}^{m}$ be the Vandermond matrix given by $v_{\alpha \beta}=\lambda_{\beta}^{\alpha-1}, \alpha, \beta=1, \cdots, r$. Then

$$
u^{(m)}=V \Lambda^{m} z \text {. }
$$

The assumption of the lemma implies that $\lim _{m \rightarrow \infty} u^{(m)}$ exists. Since $V$ is nonsingular, $\lim _{m \rightarrow \infty} \Lambda^{m} z=\lim _{m \rightarrow \infty} V^{-1} u^{(m)}$ and so $z=0$.

THEOREM 3.4. Let $A \in \mathbb{R}_{+}^{n n}$ where $\rho(A)=1$. Let $1 \leqq i, j \leqq n$.

(i) If $\lambda \in \operatorname{spec} A,|\lambda|=1$, then $\operatorname{index}_{i j}(\lambda) \leqq \operatorname{index}_{i j}(1)$.

(ii) Let q be a positive integer such that $\lambda^{q}=1$ if $\lambda \in \operatorname{spec} A,|\lambda|=1$ and index $\operatorname{x}_{i j}(\lambda)=$ 
$\operatorname{index}_{i j}(1)$. Put $k+1=\operatorname{index}_{i j}(1)$ and let

$$
B^{(m)}=A^{m}\left(I+\cdots+A^{q-1}\right) .
$$

Then $b_{j i}^{(m)} \approx m^{k}$. In particular, $a_{i j}^{(m)} \neq o\left(m^{k}\right)$ if $k \geqq 0$.

Proof. (i) Let $\left\{\lambda_{1}, \cdots, \lambda_{r}\right\}$ be the eigenvalues with $\left|\lambda_{\alpha}\right|=1, \alpha=1, \cdots, r$, where the $\lambda_{\alpha}$ are pairwise distinct. Let

$$
d+1=\max \left\{\text { index }_{i j}\left\{\lambda_{\alpha}\right\}: \alpha=1, \cdots, \dot{r}\right\} .
$$

If $d=-1$ then there is nothing to prove. So assume that $d \geqq 0$. Suppose that $z_{\alpha} \equiv z_{i j}^{(\alpha d)} \neq$ 0 for $\alpha=1, \cdots, s$ where $1 \leqq s \leqq r$ and $z_{i j}^{(\alpha d)}=0$ for $\alpha=s+1, \cdots, r$. It follows immediately from (3.1) that

$$
a_{i j}^{(m)}=m^{d}\left(\sum_{\alpha=1}^{s} \lambda_{\alpha}^{m-d} z_{\alpha}\right)+o\left(m^{d}\right)
$$

Hence, by Lemma (3.3), $a_{i j}^{(m)} \neq o\left(m^{d}\right)$.

Let $q$ be a positive integer such that $\lambda_{\alpha}^{q}=1, \alpha=1, \cdots, s$. Define

$$
\varphi_{m}(\tau)=\tau^{m}\left(1+\tau+\cdots+\tau^{q-1}\right) .
$$

If we take the $d$ th derivative of $\varphi_{m}(\tau)$, we obtain

$$
\varphi_{m}^{(d)}(\tau)=m^{d} \varphi_{m-d}(\tau)+o\left(m^{d}\right)
$$

for any fixed $\tau,|\tau| \leqq 1$, and also $\varphi_{m-d}\left(\lambda_{\alpha}\right)=0$ for $\left|\lambda_{\alpha}\right|=1, \lambda_{\alpha} \neq 1,1 \leqq \alpha \leqq s$. Put $B^{(m)}=\varphi_{m}(A)$. By (3.1) and the equality above we have

$$
b_{i j}^{(m)}=m^{d}\left(\sum_{\alpha=1}^{r} \varphi_{m-d}\left(\lambda_{\alpha}\right) z_{\alpha}\right)+o\left(m^{d}\right) .
$$

Now suppose that index ${ }_{i j}(1)<d+1$. Then (3.5) implies that $b_{i j}^{(m)}=o\left(m^{d}\right)$. But $b_{i j}^{(m)}=$ $a_{i j}^{(m)}+\cdots+a_{i j}^{(m+q-1)} \geqq a_{i j}^{(m)} \geqq 0$, and this is a contradiction. Thus $d=k$ and this proves (i).

(ii) Suppose that $\lambda_{1}=1$. If $k=-1$, by an argument like that above, $a_{i j}^{(m)}=b_{i i}^{(m)} \approx$ $m^{k}$. Let $k \geqq 0$. By (3.5) and the preceding argument we obtain

$$
b_{i j}^{(m)}=m^{k} q z_{1}+o\left(m^{k}\right),
$$

where $z_{1}=z_{i j}^{k}>0$. This proves (ii).

We now state a global version of Theorem 3.4 (ii) which follows immediately from Theorem 3.4.

THEOREM 3.6. Let $A \in \mathbb{R}_{+}^{n n}$ where $\rho(A)=1$. Let $q$ be a positive integer such that $\lambda^{a}=1$ if $\lambda \in \operatorname{spec} A,|\lambda|=1$ and index $(\lambda)=\operatorname{index}(1)=k+1$. Let

$$
B^{(m)}=A^{m}\left(I+\cdots+A^{q-1}\right) \text {. }
$$

Then

$$
\lim _{m \rightarrow \infty} m^{-k} B^{(m)}=F
$$

where $F \geqq 0$ and $F$ is not identically zero.

It should be noted that the assumption that $A$ is nonnegative was used crucially in the proof of Theorems 3.4 and 3.6. For example, let $A=-I$; then there are no $k, q$ for which the limit of (3.7) exists and is nonzero. Also, the assumption that $\rho(A)=1$ is used 
in an essential way. Let

$$
A=\left[\begin{array}{ll}
0 & 1 \\
4 & 0
\end{array}\right]
$$

Then $\lim _{m \rightarrow \infty} \rho(A)^{-2 m} A^{2 m}(I+A)$ and $\lim _{m \rightarrow \infty} \rho(A)^{-(2 m+1)} A^{2 m+1}(I+A)$ exist, but are distinct. It follows that no $k, q$ exist for which $\lim _{m \rightarrow \infty} \rho(A)^{-m} m^{-k} B^{(m)}$ exists and is nonzero.

Our subsequent work discusses the nature of $k, q$ and $F$.

4. Graph theoretical concepts. Let $A \in \mathbb{R}_{+}^{n n}$ and let $\rho(A)>0$. We may assume, without loss of generality, that after simultaneous permutations of rows and columns, $A$ is in the Frobenius [6] normal form which can be found in many references, e.g., Gantmacher [7, Vol. II, p. 75]. Thus

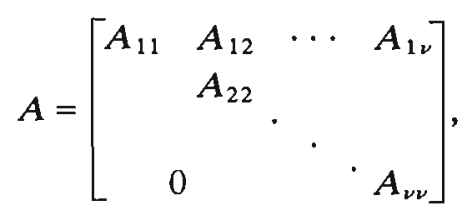

where the diagonal blocks $A_{\alpha \alpha}, \alpha=1, \cdots, \nu$ are irreducible and all subdiagonal blocks are 0 . (The $1 \times 1$ matrix 0 is considered to be irreducible.)

Let $A$ be in Frobenius normal form (4.1). Then the (reduced) graph $G(A)$ of $A$ is a subset of $\langle\nu\rangle \times\langle\nu\rangle$, where $\langle\nu\rangle=\{1, \cdots, \nu\}$ and $G(A)=\left\{(\alpha, \beta) \in\langle\nu\rangle \times\langle\nu\rangle: A_{\alpha \beta} \neq 0\right\}$. (Observe that many authors would call $G(A)$ the arcset of the graph $(\langle\nu\rangle, G(A)$ ), but we have no need to mention the vertex set $\langle\nu\rangle$ explicitly.)

If $(\alpha, \beta) \in G(A)$, we call $(\alpha, \beta)$ an arc of $G(A)$. If $(\alpha, \beta)$ is an arc of $G(A)$, then $\alpha \leqq \beta$; also $(\alpha, \alpha) \in G(A), 1 \leqq \alpha \leqq \nu$, unless $A_{\alpha \alpha}$ is the $1 \times 1$ matrix 0 . Thus we define a (simple) path from $\alpha$ to $\beta$ in $G(A)$ to be a sequence $\pi=\left(\alpha_{0}, \cdots, \alpha_{s}\right)$, where either $s \geqq 1$, $1 \leqq \alpha=\alpha_{0}<\cdots<\alpha_{s}=\beta \leqq \nu$ and $\left(\alpha_{i-1}, \alpha_{i}\right) \in G(A), i=1, \cdots, s$, or $s=0$ and $\alpha=\alpha_{0}=\beta$ and $(\alpha, \alpha) \in G(A)$. The support of $\pi$ is the set supp $\pi=\left\{\alpha_{0}, \cdots, \alpha_{s}\right\} \subseteq\{1, \cdots, \nu\}$. We always assume that the $\alpha_{i}, i=0, \cdots, s$, have been listed in strictly ascending order.

If $1 \leqq \alpha \leqq \nu$, then we call $\alpha$ a singular vertex (of $G(A)$ ) if $\rho\left(A_{\alpha \alpha}\right)=\rho(A)$. (This terminology is consistent with that of Richman-Schneider [12].) Let $1 \leqq \alpha \leqq \beta \leqq \nu$. For any path $\pi$ from $\alpha$ to $\beta$ in $G(A)$, let $k(\pi)+1$ be the number of singular $\gamma$ in the support of $\pi$. (Thus note each distinct $\gamma$ is counted only once in $k(\pi)+1$.) Let $\alpha_{j_{0}}<\alpha_{j_{1}}<\cdots<$ $\alpha_{j_{k}}$, where $k=k(\pi)$, be all singular vertices in supp $\pi$. We set

$$
k(\alpha, \beta)=\max \{k(\pi): \pi \text { is a path from } \alpha \text { to } \beta \text { in } G(A)\} .
$$

If there is no path from $\alpha$ to $\beta$ in $G(A)$ we put $k(\alpha, \beta)=-\infty$. We shall call $k(\alpha, \beta)$ the singular distance from $\alpha$ to $\beta$. If $(i, i)$ is a position in $A_{\alpha \alpha}$ and $(j, j)$ a position in $A_{\beta \beta}$ then we shall also call $k[i, j]=k(\alpha, \beta)$ the singular distance from $i$ to $j$ (note our use of square brackets).

A path $\pi$ from $\alpha$ to $\beta$ will be called a maximal path if the number of singular vertices in the support of $\pi$ is $k(\alpha, \beta)+1$. Let $1 \leqq \alpha, \beta \leqq \nu$. Let $\mathscr{P}(\alpha, \beta)$ be the set of maximal paths from $\alpha$ to $\beta$. For each $\pi \in \mathscr{P}(\alpha, \beta)$ let $q(\pi)$ be the g.c.d. of periods of $A_{\gamma \gamma}$ with $\gamma \in \operatorname{supp} \pi$ and singular (viz. $\rho\left(A_{\gamma \gamma}\right)=\rho(A)$ ).

Then we define

$$
q(\alpha, \beta)=\text { l.c.m. }\{q(\pi): \pi \in \mathscr{P}(\alpha, \beta)\} .
$$

We shall call $q(\alpha, \beta)$ the local period of $(\alpha, \beta)$. If $k(\alpha, \beta)<0$ then $q(\alpha, \beta)=1$. Also if 
$(i, i)$ is a position in $\boldsymbol{A}_{\alpha \alpha}$ and $(j, j)$ is a position in $\boldsymbol{A}_{\beta \beta}$ then we shall put $q(\alpha, \beta)=q[i, j]$, the local period of $(i, j)$.

5. The main results. Let $A \in \mathbb{R}_{+}^{n n}$, where $\rho(A)=1$, be in Frobenius normal form (4.1). It follows from the Perron-Frobenius theory for nonnegative matrices, e.g., Gantmacher [7, Chap. 13] that there is a diagonal matrix $X$ with positive diagonal elements so that, upon replacing $A$ by $X^{-1} A X$,

$$
A_{\alpha \alpha}=\rho\left(A_{\alpha \alpha}\right) A_{\alpha \alpha}^{\prime}
$$

where $A_{\alpha \alpha}^{\prime}$ is a stochastic matrix,

$$
\left\|A_{\alpha \beta}\right\|_{\infty} \leqq \sigma, \quad 1 \leqq \alpha<\beta \leqq \nu,
$$

where $1>\sigma$ and $\sigma>\max \left\{\rho\left(\boldsymbol{A}_{\alpha \alpha}\right): \rho\left(\boldsymbol{A}_{\alpha \alpha}\right)<1, \alpha=1, \cdots, \nu\right\}$ if such $\alpha$ exist. Here \|\|$_{\infty}$ is the $l_{\infty}$-operator norm,

$$
\|Z\|_{\infty}=\max \left\{\sum_{i=1}^{n}\left|z_{i j}\right|: i=1, \cdots, r\right\} \quad \text { for } Z \in \mathbb{R}^{r n} .
$$

The diagonal matrix $X$ can be constructed as follows. Let $u^{(\alpha)}$ be a positive vector satisfying $A_{\alpha \alpha} u^{(\alpha)}=\rho\left(A_{\alpha \alpha}\right) u^{(\alpha)}$. Denote by $X_{\alpha}$ a diagonal matrix, whose diagonal entries are the elements of $u^{(\alpha)}$. Then $X$ is of the form $\operatorname{diag}\left\{X_{1}, \varepsilon X_{2}, \cdots, \varepsilon^{\nu-1} X_{\nu}\right\}$ for some small enough positive $\varepsilon$. In our subsequent proofs we may assume that $A$ has been normalized as above.

Let $\pi$ be a path in $G(A)$. Denote by $s+1$ the cardinality of supp $\pi$. That is

$$
\text { supp } \pi=\left\{\beta_{0}, \cdots, \beta_{s}\right\}, \quad 1 \leqq \beta_{0}<\beta_{2}<\cdots<\beta_{s} \leqq \nu .
$$

We define the path matrix $A(\pi)$ by

$$
\begin{array}{ll}
A_{i i}(\pi)=A_{\beta_{i} \beta_{i},} & i=0, \cdots, s, \\
A_{i, i+1}(\pi)=A_{\beta_{i} \beta_{i+1}}, & i=0, \cdots, s-1, \\
A_{i j}(\pi)=0, & i, j=0, \cdots, s \text { otherwise, } \\
A(\pi)=\left(A_{i j}(\pi)\right)_{0}^{s} . &
\end{array}
$$

Thus $\boldsymbol{A}(\boldsymbol{\pi})$ is in Frobenius normal form and has $s+1$ irreducible diagonal blocks $\boldsymbol{A}_{i i}(\pi)=\boldsymbol{A}_{\boldsymbol{\beta}_{\beta} \beta_{i}}, i=0, \cdots, s$. To avoid ambiguity, we write $\boldsymbol{A}(\boldsymbol{\pi})_{i j}^{(m)}$ for the $(i, j)$ block component of $A(\pi)^{m}, i, j=0, \cdots, s$.

We now prove a sequence of lemmas for the path matrix $A(\pi)$ of a given path.

LEMMA 5.4. Let $A \in \mathbb{R}_{+}^{n n}$ where $\rho(A)=1$. Let $1 \leqq \alpha, \beta \leqq \nu$ and $\pi$ be a path in $G(A)$ from $\alpha$ to $\beta$. Put $k=k(\pi)$, where $k(\pi)+1$ is the number of singular vertices in supp $\pi$. If $A(\pi)$ is the path matrix given by (5.3), then $\left\|A(\pi)_{0 s}^{(m)}\right\|_{\infty}=O\left(m^{k}\right)$.

Proof. We note that

$$
\boldsymbol{A}(\pi)_{0 s}^{(m)}=\sum_{p_{1}+\cdots+p_{s}=m-s} \dot{A}_{00}^{p_{0}}(\pi) A_{01}(\pi) A_{11}^{p_{1}}(\pi) \cdots A_{(s-1) s}(\pi) A_{s s}^{p_{s}}(\pi) .
$$

So

$$
\left\|A(\pi)_{0 s}^{(m)}\right\|_{\infty} \leqq \sigma^{s} \sum_{p_{0}+\cdots+p_{s}=m-s}\left\|A_{00}(\pi)\right\|_{\infty}^{p_{0}} \cdots\left\|A_{s s}(\pi)\right\|_{\infty}^{p_{s}}
$$

Suppose first that $\pi$ does not contain singular vertices, i.e., $k=-1$. Then

$$
\left\|\boldsymbol{A}(\pi)_{0 s}^{(m)}\right\|_{\infty} \leqq \sigma^{m} \sum_{p_{0}+\cdots+p_{s}=m-s} 1^{p_{u}} \cdots 1^{p_{s}}=\sigma^{m} \Gamma_{s+1}^{m-s}
$$


where $\Gamma^{r}$ is given by (2.3). As $\Gamma_{s}^{m-s} \leqq m^{s}$ we immediately deduce

$$
\lim _{m \rightarrow \infty} \tau^{-m} A(\pi)_{0 s}^{(m)}=0 \quad \text { for any } \tau, \sigma<\tau<1 .
$$

Suppose now that $k \geqq 0$. Then

$$
\begin{aligned}
\left\|A(\pi)_{0 s}^{(m)}\right\|_{\infty} & \leqq \sigma^{s} \sum_{q_{0^{+}} \cdots+q_{s}=m-s} 1^{q_{0}} \cdots 1^{q_{k}} \sigma^{q_{k+1}} \cdots \sigma^{q_{s}} \\
& =\sigma^{s} \sum_{u=0}^{m-s}\left(\sum_{q_{0^{+}}+\cdots+q_{k}=u} 1^{q_{0}} \cdots 1^{q_{k}}\right)\left(\sum_{q_{k+1}+\cdots+q_{s}=m-s-u} \sigma^{q_{k+1} \cdots \sigma^{a_{s}}}\right) \\
& =\sigma^{s} \sum_{u=0}^{m-s} \Gamma_{k+1}^{u} \Gamma_{s-k}^{m-s-u} \sigma^{m-s-u} .
\end{aligned}
$$

Hence

$$
\left\|A(\pi)_{0 s}^{(m)}\right\|_{\infty} \leqq \Gamma_{k+1}^{m-s}\left(\sum_{\nu=0}^{\infty} \Gamma_{s-k}^{\nu} \sigma^{\nu+s}\right)
$$

The last series converges by the ratio test and $\Gamma_{k+1}^{m-s} \leqq m^{k}$. This establishes the lemma.

LEMMA 5.6. Let the assumptions of Lemma 5.4 hold. Assume furthermore that $k \geqq 0$, i.e., the support of $\pi$ contains singular vertices. Then, for sufficiently large $m$,

$$
\sum_{j=0}^{2(s+1)(n-1)} A(\pi)_{0 s}^{(m+j)} \geqq G m^{k}
$$

where $G$ is a positive matrix.

Proof. Let

$$
B_{i i}(\pi)=I+A_{i i}(\pi)+\cdots+A_{i i}(\pi)^{(n-1)} ; \quad i=1, \cdots, s .
$$

Since $A_{i i}(\pi)$ is irreducible, and its dimension does not exceed $n$, we have $B_{i i}(\pi)>0$, Wielandt [20], Berman-Plemmons [1, Chap. 2, Thm. 1.3]. Clearly (5.5) implies, for $t=2(s+1)(n-1)$,

$$
\begin{gathered}
\sum_{i=0}^{1} A(\pi)_{0 s}^{(m+j)} \geqq n^{-(s+1)} \sum_{p_{0}+\cdots+p_{s}=m-s} B_{00}(\pi) A_{00}(\pi)^{p_{0}} B_{00}(\pi) A_{01}(\pi) B_{11}(\pi) A_{11}^{p_{1}} B_{11}(\pi) \cdots \\
\cdot A_{s-1, s}(\pi) B_{s s}(\pi) A_{s s}^{p_{s}}(\pi) B_{s s}(\pi)
\end{gathered}
$$

For $i, j=0, \cdots, s$, let $E_{i j}$ be the matrix all of whose entries equal 1 and whose dimension is that of $A_{i j}(\pi)$. Clearly $B_{00}(\pi) \geqq c_{0}^{\prime} E_{00}, B_{s s}(\pi) \geqq c_{s}^{\prime} E_{s s}$ where $c_{0}^{\prime}, c_{s}^{\prime}>0$. Since $A_{i, i+1}(\pi) \neq 0$, we have

$$
B_{i i}(\pi) A_{i, i+1}(\pi) B_{i+1, i+1}(\pi) \geqq c_{i} E_{i, i+1},
$$

where $c_{i}>0, i=1, \cdots, s-1$, and hence, for some $c>0$,

$$
\sum_{i=1}^{l} A(\pi)_{0 s}^{(m+i)} \geqq c \sum_{p_{0}+\cdots+p_{s}=m-s} E_{00} A_{00}(\pi)^{p_{0}} E_{0}, \cdots, E_{s-1, s} A_{s s}(\pi)^{p_{*}} E_{s s}
$$

In the inequality (5.8) we may restrict the sum on the right-hand side by letting $p_{j}=0$ if $\rho\left(A_{i j}(\pi)\right)<1$. So let $\gamma_{10}<\cdots<\gamma_{k}$ be the subscripts of $A_{i i}$ which are singular vertices 
and put $\bar{A}_{i i}=A_{\gamma_{i} \gamma_{i}}(\pi)$. Since $E_{i j} E_{i k} \geqq E_{i k}$, it follows that

$$
\sum_{i=0}^{l} A(\pi)_{0 s}^{(m+i)} \geqq c^{\prime} \sum_{p_{0}+\cdots+p_{k}=m-s} \bar{E}_{-1,0} \bar{A}_{00}(\pi)^{p_{0}} \bar{E}_{01} \cdots \bar{A}_{k k}^{p_{k}} \bar{E}_{k, k+1},
$$

where $c^{\prime}>0$ and the $\bar{E}_{i, i+1}, i=-1, \cdots, k$ are matrices all of whose entries are 1 . But $\bar{A}_{i i}(\pi)$ is a stochastic matrix, $i=0, \cdots, k$, whence $\bar{A}_{i i}(\pi)^{p_{0}} \bar{E}_{i, i+1}=\bar{E}_{i, i+1}, i=0, \cdots, k$. It follows that

$$
\sum_{i=0}^{l} A(\pi)_{0 s}^{(m+i)} \geqq 2 \Gamma_{k+1}^{m-s} G,
$$

where $G>0$. The lemma now follows from (2.3) since $\Gamma_{k}^{m-s} \geqq \frac{1}{2} m^{k}$ for sufficiently large $m$.

LEMMA 5.9. Let the assumptions of Lemma 5.4 hold, and suppose that $k=k(\pi) \geqq 0$. Let $q=q(\pi)$ be the g.c.d. of periods of $A_{\gamma \gamma}$ for singular $\gamma \in \operatorname{supp} \pi$. Let

$$
B(\pi)^{(m)}=A(\pi)^{m}\left(I+A(\pi)+\cdots+A(\pi)^{q-1}\right) .
$$

(i) If $(i, j)$ is any position in $A(\pi)_{0 s}$ then, in $A(\pi)$, index $i j(1)=k+1$.

(ii) $b(\pi)_{i j}^{(m)} \approx m^{k}$.

Proof. (i) Let $k^{*}+1=$ index $_{i j}$ (1) in $A(\pi)$. By Theorem 3.4 there is a positive integer $q^{*}$ such that for

$$
B^{*}(\pi)^{(m)}=A(\pi)^{m}\left(I+A(\pi)+\cdots+A(\pi)^{q^{*-1}}\right),
$$

we have $b^{*}(\pi)_{i j .}^{(m)} \approx m^{k^{*}}$. But $k^{*}>k$ contradicts Lemma 5.4. Since the sum in (5.7) can be majorized by a sum of terms of the form $B^{*}(\pi)_{0 s}^{(m+j)}, j=0, \cdots, 2(s+1)(n-1)$, it follows that $k^{*}<k$ contradicts Lemma 5.6. Hence $k^{*}=k$.

(ii) Now suppose that $\lambda \in \operatorname{spec} A(\pi),|\lambda|=1$ and $\operatorname{index}_{i j}(\lambda)=\operatorname{index}_{i j}(1)=k+1$ in $A(\pi)$. Then

$$
\operatorname{index}_{i j}(\lambda) \leqq \operatorname{index}(\lambda) \leqq \operatorname{mult}(\lambda),
$$

where mult $(\lambda)$ is the algebraic multiplicity of $\lambda$ in $A(\pi)$. But, by the Perron-Frobenius theorem for irreducible matrices,

$$
\operatorname{mult}(\lambda) \leqq \operatorname{mult}(1)=k+1 \text {. }
$$

Hence mult $(\lambda)=k+1$ and, by Perron-Frobenius, $\lambda$ is an eigenvalue of every $A_{\gamma \gamma}$ for which $\gamma$ is singular. It follows that $\lambda^{a}=1$, where $q=q(\pi)$. Hence the conditions of Theorem 3.4 (ii) are satisfied and the lemma follows.

We state our main result.

THEOREM 5.10. Let $A$ be nonzero $n \times n$ matrix normalized by the condition $\rho(A)=1$. Assume $1 \leqq i, j \leqq n$. Let $k=k[i, j]$ be the singular distance from $i$ to $j$ and $q=q[i, j]$ be the local period of $(i, j)$. Put $B^{(m)}=A^{m}\left(I+A+\cdots+A^{q-1}\right)$. Then $b_{i j}^{(m)} \approx$ $m^{k}$.

Proof. As usual, we assume that $A$ is in the Frobenius form (4.1). Suppose that $(i, j)$ is a position in $A_{\alpha \beta}$. Denote by $\Pi(\alpha, \beta)$ the set of all paths connecting $\alpha$ to $\beta$. Then we obviously have

$$
A_{\alpha \beta}^{(m)}=\sum_{\pi \in \Pi(\alpha, \beta)} A(\pi)_{O s(\pi)}^{(m)}
$$

So

$$
B_{\alpha \beta}^{(m)}=\sum_{\pi \in \Pi(\alpha, \beta)} B(\pi)_{() s(\pi)}^{(m)}
$$


Assume first that $k=k(\pi)=-\infty$; then, clearly, $B_{\alpha \beta}^{(m)}=A_{\alpha \beta}^{(m)}=0$. If $k=-1 \geqq k(\pi)$ then Lemma 5.4 implies that each $A(\pi)_{0 s(\pi)}^{(m)} \approx m^{-1}$. So $A_{\alpha \beta}^{(m)} \approx m^{-1}$ and again $A_{\alpha \beta}^{(m)}=B_{\alpha \beta}^{(m)}$.

Assume now that $k \geqq 0$. If $k>k(\pi)$, Lemma 5.4 implies that $B(\pi)_{(j)(\pi)}^{(m)}=O\left(m^{k}\right)$. However, if $k=k(\pi)$, then according to Lemma $5.9 \lim _{m \rightarrow \infty} m^{-k} B(\pi)_{0 s(\pi)}^{(m)}=F_{0 s}(\pi)>0$ as $q(\pi)$ divides $q(\alpha, \beta)=q[i, j]$. By the definition of $k(\alpha, \beta)$ there exists $\pi \in \Pi(\alpha, \beta)$ such that $k(\pi)=k(\alpha, \beta)$. So $\lim _{k \rightarrow \infty} m^{-k} B_{\alpha \beta}^{(m)}=F_{\alpha \beta}>0$.

COROLlaRY 5.11. Under the conditions of Theorem 5.10,

$$
\sum_{p=1}^{m} a_{i j}^{(p)} \approx m^{(k+1)}
$$

Proof. For $k \geqq 0$, the result is immediate by Lemma 2.4. If $k=-1$, then by Theorem 5.10 the nonnegative series above converges. The assumption $k=-1$ implies that at least one term is positive. Finally if $k=-\infty, a_{i j}^{(p)}=0, p=1,2, \cdots$, and the result follows.

Comparing Theorems 3.4 and 5.10 we first deduce a local version of Rothblum's equality and then the equality itself.

TheOREM 5.12. Let $A \in \mathbb{R}_{+}^{n n}$ where $\rho(A)=1$. Assume that $1 \leqq i, j \leqq n$; then

$$
\operatorname{index}_{i j}(1)=k[i, j]+1 \text {. }
$$

Corollary 5.13 (Rothblum [13]). Let $A \in \mathbb{R}_{+}^{n n}$ where $\rho(A)=1$. Then index $(1)=$ $\max _{1 \leq i, j \leqq n} \operatorname{index}_{i j}(1)=\max _{1 \unlhd i, j \leq n} k[i, j]+1$.

6. Convergent iterative methods for the spectral radius of a nonnegative matrix. Let $A \in \mathbb{R}_{+}^{n n}$ and assume that $\rho(A)>0$. Let $r(x)$ and $R(x)$ be defined as in (1.4). Clearly $0 \leqq r(x) \leqq R(x) \leqq+\infty$. It is obvious that

$$
r(x) \leqq r(A x) \leqq R(A x) \leqq R(x)
$$

So the sequence $r\left(A^{m} x\right), m=0,1, \cdots$ is an increasing sequence bounded above- by: $R(x)$, and the sequence $R\left(A^{m} x\right), m=0,1, \cdots$ is a decreasing sequence bounded below by $r(x)$ :

In [4], Collatz observed that, for $A \in \mathbb{R}_{+}^{n n}$ and $x>0$,

$$
r(x) \leqq \rho(A) \leqq R(x),
$$

and when $A$ is irreducible, this inequality is valid for all $x \geqq 0, x \neq 0$; see Wielandt [20], Varga $[19$, p. 32]. Thus the question arises when, for $A \geqq 0$ and $x \geqq 0, x \neq 0$,

$$
\lim _{m \rightarrow \infty} r\left(A^{m} x\right)=\rho(A)=\lim _{m \rightarrow \infty} R\left(A^{m} x\right) .
$$

Wielandt's [20] characterization of $\rho(A)$ for irreducible $A$ easily implies that (6.2) holds for primitive $A$ and all $x \in \mathbb{R}_{+}^{n}, x \geqq 0, x \neq 0$ (cf. Varga [19, p. 34]). This result follows from the fact that

$$
\lim _{m \rightarrow \infty} \rho(A)^{-m} A^{m}=Z>0
$$

when $A$ is primitive, where $Z=u v^{\prime}, v>0, A u=\rho(A) u, v>0, v^{t} A=\rho(A) v^{\prime}, v^{\prime} u=1$. If $A$ is irreducible but imprimitive then (6.2) does not hold unless $x$ is orthogonal on all eigenvectors of $A^{\prime}$ corresponding to $\lambda$ such that $|\lambda|=\rho(A)$ and $\lambda \neq \rho(A)$. We shall show 
that this condition can be put in equivalent forms. If $A$ is irreducible and of period $q$, then by simultaneous permutations of rows and columns we now put $A$ into the form

$$
\left[\begin{array}{ccccc}
0 & A_{12} & 0 & \cdots & 0 \\
0 & 0 & A_{23} & \cdots & 0 \\
\vdots & & & & \\
0 & 0 & 0 & \cdots & A_{q-1, q} \\
A_{q 1} & 0 & 0 & \cdots & 0
\end{array}\right]
$$

where the diagonal blocks 0 are square (see Frobenius [6], Gantmacher [7, Vol II, p. 62], Berman-Plemmons [1, Chap. 2, Thm. 2.20]).

LEMMA 6.4. Let $A$ be an irreducible nonnegative matrix of period $q$ in form (6.3), and suppose that $\rho(A)=1$. Let $v^{\prime} A=v^{\prime}, A u=u$, where $v>0, u>0, v^{\prime} u=1, A^{i} y^{j}=\omega^{j} y^{\prime}$, $j=1, \cdots, q-1, \omega=e^{2 \pi i / q}$. Let $0 \neq x \in \mathbb{R}_{+}^{n}$ be partitioned conformally with $A, x^{i}=$ $\left(x_{(1)}^{\prime}, \cdots, x_{(q)}^{\prime}\right)$. Then the following are equivalent

(i) $\lim _{m \rightarrow \infty} A^{m} x=\left(v^{t} x\right) u$,

(ii) $\lim _{m \rightarrow \infty} A^{m} x$ exists,

(iii) $\quad x^{i} y^{j}=0, \quad j=1, \cdots, q-1$,

(iv) $v_{(1)}^{\prime} x_{(1)}=\cdots=v_{(q)}^{\prime} x_{(q)}$,

(v) $\quad \lim _{m \rightarrow \infty} R\left(A^{m} x\right)=\lim _{m \rightarrow \infty} r\left(A^{m} x\right)=1$, where $v^{\prime}=\left(v_{(1)}^{t}, \cdots, v_{(q)}^{\prime}\right)$ has been partitioned conformally with $A$.

Proof. We first derive a formula for $A^{m} x, m=1,2, \cdots$. Let $\omega$ be a primitive qth root of unity. It is well-known that the eigenvalues of $A$ on the unit circle are $\lambda_{\alpha}=\omega^{\alpha-1}$, $\alpha=1, \cdots, q$ and that each $\lambda_{\alpha}$ is a simple zero of the characteristic polynomial. It follows, in the notation of $\S 3$, that $p_{\alpha}=0, \alpha=1, \cdots, q$ and that

$$
\begin{aligned}
& Z^{(\alpha 0)}=D^{\alpha-1} u v^{\prime} D^{1-\alpha}, \quad \alpha=1, \cdots, q, \\
& y^{\alpha}=D^{(1-\alpha)} v, \quad \alpha=1, \cdots, q,
\end{aligned}
$$

where

$$
D=\left[\begin{array}{cccc}
I_{11} & & & \\
& & \omega I_{22} & 0 \\
& 0 & \ddots & \\
& & & \omega^{q-1} I_{q q}
\end{array}\right],
$$

and $I_{\alpha \alpha}$ is an identity matrix of the same order of $A_{\alpha \alpha}, \alpha=1, \cdots, q$.

Hence by (3.1),

$$
A^{m}=\sum_{\alpha=0}^{q-1} \omega^{m \alpha} D^{\alpha} u v^{t} D^{-\alpha}+o(1)
$$

and so

$$
A^{m} x=\sum_{\alpha=0}^{q-1} \omega^{m \alpha} a_{\alpha}\left(D^{\alpha} u\right)+o(1)
$$

where

$$
a_{\alpha}=v^{t} D^{-\alpha} x=x^{t} y^{\alpha}, \quad \alpha=0, \cdots, q-1
$$

Let

$$
c_{\beta}=v_{(\beta+1)}^{\prime} x_{(\beta+1)}, \quad \beta=0, \cdots, q-1 .
$$


Then it follows immediately from $(6.6)$ that

$$
a_{\alpha}=\sum_{\beta=0}^{q-1} \omega^{-\alpha \beta} c_{\beta}, \quad \alpha=0, \cdots, q-1 .
$$

We now prove the equivalence of our five conditions. We show (i) $\Rightarrow$ (ii) $\Rightarrow$ (iii) $\Rightarrow$. (iv) $\Rightarrow$ (i) and (i) $\Rightarrow$ (v) $\Rightarrow$ (iv).

(i) $\Rightarrow$ (ii). Trivial.

(ii) $\Rightarrow$ (iii). Since $\lim _{m \rightarrow \infty} A^{m} x$ exists, $\lim _{m \rightarrow \infty} v^{\prime} D^{-\alpha} A^{m} x$ also exists, $\alpha=$ $0, \cdots, q-1$. But $v^{t} u>0$, and hence $a_{\alpha}=x^{t} y^{\alpha}=0, \alpha=1, \cdots, q-1$ by Lemma 3.3.

(iii) $\Rightarrow$ (iv). Consider the identity (6.7). Since the Vandermonde matrix $q^{-1 / 2}\left(\omega^{-\alpha \beta}\right), \quad \alpha, \beta=0, \cdots, q-1$ is unitary the assumption $a_{\alpha}=x^{8} y^{\alpha}=0, \alpha=$ $1, \cdots, q-1$ implies that $c_{0}=c_{1}=\cdots=c_{q-1}$, which proves (iv).

(iv) $\Rightarrow$ (i). If (iv) holds, then $c_{0}=c_{1}=\cdots=c_{q-1}$ and (6.7) implies $a_{1}=\cdots=a_{q-1}=0$. This establishes (i) in view of (6.5) and (6.6).

(i) $\Rightarrow(v)$. Trivial, since $v^{\prime} x>0$ and $u>0$.

(v) $\Rightarrow$ (i). Let $m=q l+r, 0 \leqq r \leqq q-1$. Then (6.5) implies

$$
\lim _{l \rightarrow \infty} A^{q l+r} x=\tilde{x}^{(r)}, \quad r=0, \cdots, q-1
$$

for some $\cdot \tilde{x}^{(r)} \geqq 0, \tilde{x}^{(r)} \not 0$. Also

$$
A^{r} \tilde{x}^{(0)}=\tilde{x}^{(r)}, \quad r=0, \cdots, q-1, \quad A^{q} \tilde{x}^{(0)}=\tilde{x}^{(0)} .
$$

As $A^{q}$ is a direct sum of $q$ irreducible and primitive matrices the assumption $x \geqq 0, x \neq 0$ implies that $\lim _{l \rightarrow \infty}\left(A^{q}\right)^{l} x=\tilde{x}^{(0)} \neq 0$. Obviously $x^{(0)} \geqq 0$.

Now $(v)$ implies that

$$
x^{0} \leqq x^{(1)}=A x^{0} \leqq x^{(0)},
$$

whence $x^{(1)}=x^{(0)}$ and thus $x^{(r)}=x^{(0)}$ for $r=1, \cdots, q-1$. So $\lim _{m \rightarrow \infty} A^{m} x=x^{(0)}$ and (i) follows.

In what follows, we give necessary and sufficient conditions on a reducible matrix $\boldsymbol{A}$ to satisfy (6.2). To do so we need a few more graph theoretical concepts.

Let $G$ be a graph on $\langle\nu\rangle=\{1, \cdots, \nu\}$. Let $J$ be a nonvoid subset of $\langle\nu\rangle$. Then $\alpha \in J$ is called a final state with respect to $J$ if for any $\beta \neq \alpha$ and $(\alpha, \beta\rangle \in G, \beta \notin J$. Denoting by $\mathscr{F}(J)$ the set of all final states with respect to $J$. If $J=\langle\nu\rangle$, then $\alpha$ is called a final state, i.e., $(\alpha, \beta) \in G$ implies that $\beta=\alpha$. Define

$$
d(\beta, J)=\max \{k(\beta, \alpha): \alpha \in \mathscr{F}(J)\} .
$$

If $J=\langle\nu\rangle$, then write $d(\beta)$ instead of $d(\beta,\langle\nu\rangle)$. Let $A \geqq 0$ be a reducible matrix. We assume that $A$ is in the Frobenius form (4.1).

As in $\S 4$, denote by $G(A)$ the (reduced) graph of $A$. Let $x \geqq 0, x \neq 0$. Partition $x$ conformably with $A$ given by (4.1). That is $x^{\prime}=\left(x_{(1)}^{\prime}, \cdots, x_{(\nu)}^{\prime}\right)$. The support of $x$ is the set supp $x=\left\{\alpha_{1}, \cdots, \alpha_{s}\right\} \subseteq\{1, \cdots, \nu\}$ such that $x_{(i)} \neq 0$ if and only if $i \in \operatorname{supp} x$. We shall always assume that $\alpha_{i}, i=1, \cdots, s$ have been listed in strictly ascending order.

THEOREM 6.8. Let $A \in \mathbb{R}_{+}^{n n}, \rho(A)>0$. Assume that $A$ is in the Frobenius form (4.1). Moreover, if $A_{i i}$ is imprimitive then $A_{i i}$ is the Frobenius form (6.3). Let $x \geqq 0, x \neq 0$. Then (6.2) holds if and only if any final state $\alpha$ with respect to the support of $x$ satisfies

(i) $\alpha$ is a singular vertex (i.e., $\rho\left(\boldsymbol{A}_{\alpha \alpha}\right)=\rho(\boldsymbol{A})$ ),

(ii) either $\boldsymbol{A}_{\alpha \alpha}$ is primitive, or $\boldsymbol{A}_{\alpha \alpha}$ and $x_{(\alpha)}$ satisfy the condition (iv) of Lemma 6.4. 
Proof. Without loss of generality we may assume that $\rho(A)=1$. Next we note that

$$
\left(A^{m} x\right)_{\alpha}=\sum_{\beta \in \text { supp } x} A_{\alpha \beta}^{(m)} x_{(\beta)} .
$$

Suppose that $\alpha \in \mathscr{F}(\operatorname{supp} x)$. Then

$$
\left(A^{m} x\right)_{\alpha}=A_{\alpha \alpha}^{m} x_{(\alpha)} .
$$

By the definition of $R(x)$ and $r(x)$ we have

$$
r\left(A^{m} x\right) A^{m} x \leqq A^{m+1} x \leqq R\left(A^{m} x\right) A^{m} x
$$

So

$$
r\left(A^{m} x\right) A_{\alpha \alpha}^{m} x_{(\alpha)} \leqq A_{\alpha \alpha}^{m+1} x_{(\alpha)} \leqq R\left(A^{m} x\right) A_{\alpha \alpha}^{m} x_{(\alpha)} .
$$

Hence, since $A_{\alpha \alpha}$ is irreducible, by (6.1),

$$
r\left(A^{m} x\right) \leqq r\left(A_{\alpha \alpha}^{m} x_{(\dot{\alpha})}\right) \leqq \rho\left(A_{\alpha \alpha}\right) \leqq R\left(A_{\alpha \alpha}^{m} x_{(\alpha)}\right) \leqq R\left(A^{m} x\right) .
$$

Assume now that (6.2) holds. Then for any final state $\alpha$ with respect to supp $x$, we must have

$$
\lim _{m \rightarrow \infty} r\left(A_{\alpha \alpha}^{m} x_{(\alpha)}\right)=\lim _{m \rightarrow \infty} R\left(A_{\alpha \alpha}^{m} x_{(\alpha)}\right)=\rho\left(A_{\alpha \alpha}\right)=1 .
$$

So $\alpha$ is a singular vertex. If $A_{\alpha \alpha}$ is imprimitive, then the condition (v) of Lemma 6.4 holds. Hence, $A_{\alpha \alpha}$ and $x_{(\alpha)}$ satisfy (iv) of Lemma 6.4. This proves one direction of our theorem.

Assume now that if $\alpha \in \mathscr{F}(\operatorname{supp} x)$ then $\rho\left(A_{\alpha \alpha}\right)=1$; and if $\boldsymbol{A}_{\alpha \alpha}$ is not primitive then $A_{\alpha \alpha}$ and $x_{(\alpha)}$ satisfy the condition (iv) of Lemma 6.4.

Let $1 \leqq \beta \leqq \nu$. Let $d=d(\beta, J)$. By our assumption, $d \neq-1$. If $d=-\infty$, then $\left(A^{m} x\right)_{\beta}=0, m=1,2, \cdots$. If $d \geqq 0$, then

$$
m^{-d}\left(A^{m} x\right)_{\beta}=m^{-d} \sum_{\alpha \in K} A_{\beta \alpha}^{(m)} x_{\alpha}+o(1),
$$

where $K=\{\alpha: k(\beta, \alpha)=d\}$. Clearly $K \subseteq \mathscr{F}(\operatorname{supp} x)$. Thus, to show

$$
\lim _{m \rightarrow \infty} m^{-d}\left(A^{m} x\right)_{\beta}>0
$$

it is enough to prove

$$
m^{-d} A_{\beta \alpha}^{(m)} x_{\alpha}>0
$$

for $\alpha \in \mathscr{F}(\operatorname{supp} x), k(\beta, \alpha)=d$. To prove (6.11), let $D$ be the matrix obtained from $A$ by setting $D_{\alpha \alpha}=0$ and $D_{\gamma \delta}=A_{\gamma \delta}$ in all other cases, $1 \leqq \gamma, \delta \leqq \nu$. We then have

$$
m^{-d} A_{\beta \alpha}^{(m)} x_{\alpha}=m^{-d} \sum_{p=0}^{m} D_{\beta \alpha}^{(m-p)} A_{\alpha \alpha}^{p} x_{\alpha} .
$$

Since in $D$, the singular distance from $\beta$ to $\alpha$ is $d-1$, we have, by Corollary 5.11,

$$
\lim _{m \rightarrow \infty} m^{-\alpha} \sum_{p=0}^{m} D_{\beta \alpha}^{(m-p)}=U_{\beta \alpha}>0
$$

and by Lemma (6.4)

$$
\lim _{p \rightarrow \infty} A_{\alpha \alpha}^{p} x_{\alpha}=v_{\alpha}>0 .
$$


It easily follows from Lemma 2.7 that

$$
\lim _{m \rightarrow \infty} m^{-d} A_{\beta \alpha}^{(m)} x_{\alpha}=\frac{1}{d} U_{\beta \alpha} v_{\alpha}>0
$$

Thus, for each $\beta, 1 \leqq \beta \leqq \nu$, either $\left(A^{m} x\right)_{\beta}=0, m=1,2, \cdots$ or $(6.10)$ is satisfied. From this $(6.2)$ follows immediately.

Corollary 6.12. Let $A \in \mathbb{P}_{+}^{n n}, \rho(A)>0$. Assume that $A$ is the Frobenius form (4.1). Let $J$ be an nonempty set of $\langle\nu\rangle$. Then for any $x \geqq 0$ whose support is the set $J,(6.2)$ holds if and only if for all final states $\alpha$ with respect to $J, \rho\left(A_{\alpha \alpha}\right)=\rho(A)$ and $A_{\alpha \alpha}$ is primitive.

Corollary 6.13. Let $A \in \mathbb{R}_{+}^{n n}, \rho(A)>0$. Assume that $A$ is in the Frobenius form (4.1). Then for any $x \geqq 0, x \neq 0,(6.2)$ holds if and only if for each $\alpha, \alpha=1, \cdots, \nu$, $\rho\left(A_{\alpha \alpha}\right)=\rho(A)$ and $A_{\alpha \alpha}$ is primitive.

7. Nonnegative solutions of $(\boldsymbol{I}-\boldsymbol{A}) \boldsymbol{y}=\boldsymbol{x}$. As an application of our results we give a simple proof of a theorem concerning nonnegative solutions $y$ of $(I-A) y=x$ for given $x \geqq 0$. For $1 \leqq \alpha, \beta \leqq \nu$ we shall say that $\beta$ has access to $\alpha$ in $G(A)$ if there is a path from $\beta$ to $\alpha$ in $G(A)$, viz., $k(\beta, \alpha) \geqq-1$.

THEOREM 7.1. Let $A \in \mathbb{R}_{+}^{n n}$ with $\rho(A)=1$, and suppose that $A$ is in the Frobenius normal form (4.1). Let $x \in \mathbb{R}_{+}^{n}$. Then the following are equivalent:

(i) there is a $y \in \mathbb{R}_{+}^{n}$ such that $(I-A) y=x$;

(ii) no singular vertex $\beta$ has access in $G(A)$ to any $\alpha \in \operatorname{supp} x$;

(iii) $\lim _{m \rightarrow \infty}\left(I+\cdots+A^{m}\right) x$ exists;

(iv) $\lim _{m \rightarrow \infty} A^{m} x=0$.

Further, if (iii) holds and $y=\lim _{m \rightarrow \infty}\left(I+A+\cdots+A^{m}\right) x$, then $(I-A) y=x$ and

$$
\begin{aligned}
& y_{\beta}=0 \quad \text { if } \beta \text { does not have access to any } \alpha \in \operatorname{supp} x, \\
& y_{\beta}>0 \quad \text { if } \beta \text { has access to some } \alpha \in \operatorname{supp} x .
\end{aligned}
$$

Proof. Let $S^{(m)}=I+A+\cdots+A^{m}$. If $1 \leqq \beta \leqq \nu$, then

$$
\left(S^{(m)} x\right)_{\beta}=\sum_{\alpha \in \operatorname{supp} x} S_{\beta \alpha}^{(m)} x_{\alpha}
$$

and, by Corollary 5.11 , for $k=k(\beta, \alpha) \geqq-1$,

$$
\lim _{m \rightarrow \infty} m^{-(k+1)} S_{\beta \alpha}^{(m)}=U_{\beta \alpha}>0
$$

while for $k(\beta, \alpha)=-\infty$,

$$
S_{\beta \alpha}^{(m)}=U_{\beta \alpha}=0, \quad m=1,2,3, \cdots .
$$

We shall prove (i) $\Rightarrow$ (ii) $\Rightarrow$ (iii) $\Rightarrow$ (i), (iii) $\Rightarrow$ (iv) $\Rightarrow$ (ii).

(i) $\Rightarrow$ (ii). Suppose that $(I-A) y=x$, where $y \geqq 0$. Then

$$
S^{(m)} x=\left(I-A^{m+1}\right) y \leqq y .
$$

Let $\beta$ be a singular vertex. If $\beta$ has access to $\alpha$, then $k=k(\beta, \alpha) \geqq 0$ and, by (7.4) and (7.5),

$$
y_{\beta} \geqq\left(S^{(m)} x\right)_{\beta} \geqq \frac{1}{2} m^{(k+1)} U_{\beta \alpha} x_{\alpha}
$$

for large $m$. Hence $x_{\alpha}=0$ and $\alpha \notin \operatorname{supp} x$.

(ii) $\Rightarrow$ (iii). Suppose (ii) holds and let $1 \leqq \beta \leqq \alpha$. 
If $\alpha \in \operatorname{supp} x$, then $k=k(\beta, \alpha)=-1$, or $k=-\infty$. Hence, $\lim _{m \rightarrow \infty} S_{\beta \alpha}^{(m)} x_{\alpha}=U_{\beta \alpha} x_{\alpha}$ exists for $\alpha \in \operatorname{supp} x_{\alpha}$. So, by (7.5), $\lim _{m \rightarrow \infty} S^{(m)} x$ exists.

(iii) $\Rightarrow$ (i). Let $y=\lim _{m \rightarrow \infty} S^{(m)} x$. Clearly $y \geqq 0$. Since $A S^{(m)} x=S^{(m+1)} x-x, y$ satisfies $(I-A) y=x$. This proves (i).

(iii) $\Rightarrow$ (iv). Trivial.

(iv) $\Rightarrow$ (ii). Suppose that (iv) holds but that (ii) is false. Then there exists a singular $\beta$ and an $\alpha \in \operatorname{supp} x$. such that $k(\beta, \alpha) \geqslant 0$. Let $q=q(\beta, \alpha)$ be the local period and let $B^{(m)}=A^{m}\left(I+\cdots+A^{q-1}\right)$. Then $\lim _{m \rightarrow \infty} B^{(m)} x=0$. But by Theorem 5.10 for all sufficiently large $m$,

$$
\left(B^{(m)} x\right)_{\beta} \geqq B_{\beta \alpha}^{(m)} x_{\alpha} \geqq c m^{k} x_{\alpha}
$$

where $c>0$, and $x_{\alpha} \neq 0$. This is a contradiction, and the implication is proved.

To complete the proof of the theorem observe that, for $y=\lim _{m \rightarrow \infty} S^{(m)} x$,

$$
y_{\beta}=\sum_{\alpha \in \operatorname{supp} x} U_{\beta \alpha} x_{\alpha}
$$

in view of (ii) and (7.5). Since $U_{\beta \alpha}>0$, if $\beta$ has access to $\alpha$ and $U_{\beta \alpha}=0$ otherwise, we immediately obtain (7.2) and (7.3).

The equivalence of conditions (i) and (ii) in Theorem 7.1 is due to D. H. Carlson [3]. We remark that Carlson also showed that if a nonnegative solution $y$ of $(I-A) y=x$ exists, then the solution satisfying (7.2) and (7.3) is unique. It should be observed that the assumption that $A$ is in Frobenius normal form is not needed for conditions (i), (iii) and (iv) of Theorem 7.1, which may easily be proved equivalent directly. Conditions (iii) and (iv) are equivalent for general $A \in \mathbb{R}^{n n}$ and $x \in \mathbb{R}^{n}$. We observe that for

$$
A=\left[\begin{array}{ll}
1 & 1 \\
0 & 1
\end{array}\right], \quad x=\left[\begin{array}{l}
1 \\
0
\end{array}\right]
$$

there is a $y \in \mathbb{R}^{n}$ such that $(I-A) y=x$; yet the equivalent conditions (ii), (iii) and (iv) do not hold. Clearly, no $y$ satisfying $(I-A) y=x$ can be nonnegative.

\section{REFERENCES}

[1] A. Berman And R. J. Plemmons, Nonnegative matrices in the mathematical sciences, Academic Press, New York, 1979.

[2] R. A. BRualdi, Introductory Combinatorics, North Holland, Amsterdam, 1977.

[3] D. H. CARLson, A note on M-matrix equations, J. Soc. Indust. Appl. Math., 11 (1963), pp. 1027-1033.

[4] L. CollatZ, Einschliessungssatz für die charakteristischen Zahlen von Matrizen, Math. Z., 48 (1942), pp. 221-226.

[5] S. FRIEDLAND, On an inverse problem for nonnegative matrices and eventwally nonnegative matrices, Israel J. Math., 29 (1978), pp. 43-60.

[6] G. F. Frobenius, Über Matrizen aus nicht negativen Elementen, S. B. Kön. Preuss. Akad. Wiss. Berlin, (1912), pp. 456-477; Gesammelte Abhandlungen, vol. 3, Springer, Berlin, 1968, pp. 546-567.

[7] F. R. GANTMACHER, The Theory of Matrices, Chelsea, New York, 1959.

[8] G. H. HARDY, Divergent Series, Clarendon, Oxford, England, 1949.

[9] S. KARLIN, Positive operators, J. Math. Mech., 8 (1959), pp. 907-937.

[10] C. D. MEYER AND R. J. PLEMMONS, Convergent powers of a matrix with applications to iterative methods for singular systems, SIAM J. Numer. Anal, 14 (1977), pp. 699-705.

[11] A. Ostrowsk1, Über die Determinanten mit übenwiegender Hauptdiagonale, Comment. Math. Helv., 10 (1937), pp. 69-96.

[12] D. RICHMAN AND H. SCHNEIDER, On the singular graph and the Weyr characteristic of an $M$-matrix, Aequationes Math., 17 (1978), pp. 208-234.

[13] U. G. RотнвLUM, Algebraic eigenspaces of nonnegative matrices, Linear Algebra and Appl., 12 (1975), pp. 281-292. 
[14] U. G. RотнвLuм, Expansions of sums of matrix powers and resolvents, Yale Univ. Rep., New Haven, CT, revised Jan. 1977: S1AM Rev., to appear.

[15] - Sensitive growth analysis of multiplicative systems $I$ : The dynamic approach. Yale Univ. Rep.. New Haven, CT, revised Jan. 1977.

[16] H. H. SCHAEFER, Topological Vector Spaces, Macmillan, New York, 1964.

[17] H. H. SChAEFER, Banach Lattices and Positive Operators, Springer, New York, 1974.

[18] E. C. Titchmarsh, The Theory of Functions, 2nd ed., Oxford University Press, London, 1939.

[19] R. S. VARGA, Matrix Iierative Analysis, Prentice Hall, Englewood Cliffs, NJ, 1962.

[20] H. WIELANDT, Unzerlegbare, nicht negative Matrizen, Math. Z., 52 (1950), pp. 642-648. 